\title{
Super Refractory Status Epilepticus in a Case of Post Mumps Autoimmune Encephalitis: A Rare Case Report
}

\section{Satabdi Giri, Priyanka Agarwal, Mamta Kumari and Dillip Kumar Dash}

Department of Paediatrics, IMS \& SUM Hospital, Bhubaneswar, India

\section{Correspondence: \\ Satabdi Giri \\ Department of paediatrics \\ IMS \& SUM Hospital, \\ Bhubaneswar, India \\ E-mail: rumu1406@gmail.com}

DOI: $10.3126 /$ jnps.v39i2.27862

Submitted on: $2020-02-26$

Accepted on: 2020-03-15

Acknowledgements: None

Funding: Nil

Conflict of Interest: None declared

Permission form IRB: Yes

To cite this article: Giri S, Agarwal P, Kumari M, Dash DK. Super refractory status epilepticus in a case of post mumps autoimmune encephalitis: A rare case report. J Nepal Paediatr Soc. 2019;39(2): $127-30$.

\section{ABSTRACT}

Seizure is a common problem in many paediatric patients. When seizures persist even after 24 hrs of anaesthetic treatment it is known as Super Refractory Status Epilepticus (SRSE). We report a 10 years old girl admitted with fever, altered sensorium and GTCS with a provisional diagnosis of viral encephalitis. In the line of SRSE infusion thiopental was also started after giving first line antiepileptic as per guidelines. Seizures persisted despite six anti-epileptic drugs, steroids and infusion anaesthetic. MRI brain was suggestive of T2W Flair hyperintensity in $\mathrm{B} / \mathrm{L}$ hippocampal and Para-hippocampal gyrus and CSF and blood positive for Mumps IgM. This was suggestive of Mesial temporal sclerosis (MTS) which is the scarring and loss of neurons in the deepest portion of the temporal lobe due to oxygen starvation to the brain, head trauma, brain infection or without any apparent cause. Dramatic response was seen after 36-48 hours of IVIG administration.

Key words: Mesial Temporal Sclerosis; Mumps Encephalitis; Super Refractory Status Epilepticus (SRSE)

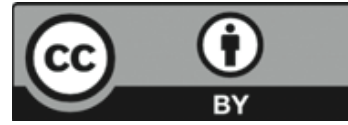

This work is licensed under creative common attribution 3.0 license 


\section{INTRODUCTION}

Paediatric patients presenting with an encephalitic syndrome represent a diagnostic challenge for evaluation and appropriate treatment. Autoimmune conditions are not a common cause of encephalitis. However, with recognition of many causative antibodies, diagnostic serology is becoming increasingly available and used for diagnosis. Mesial temporal sclerosis (MTS) is the scarring and loss of neurons in the deepest portion of the temporal lobe due to oxygen starvation to the brain, head trauma, brain infection or without any apparent cause. The left and right medial temporal lobes are most commonly affected which include the hippocampus and amygdala. Scarring of the hippocampus is the commonest form and is known as hippocampal sclerosis. Symptoms of mesial temporal sclerosis are generally associated with focal (partial) epilepsy. ${ }^{1}$ Seizures that persist even after giving two antiepileptic drugs ( $1^{\text {st }}$ line being a BZD) is said to be refractory status epilepticus. But if seizures persist even after 24 hours of anesthetic infusion or recurrence of seizure on tapering of anesthetic infusion, is said to be super-refractory status epilepticus. ${ }^{2}$

\section{CASE REPORT}

We report a 10 years old female child from a small village Khurdha, India who was admitted to our hospital with chief complaints of fever, headache for four days and altered sensorium for one day (Figure. 3). The child had history of mumps parotitis two weeks back. On examination meningeal signs were negative and bilateral pupils were reacting to light. On admission to PICU, child had an episode of GTCS lasting for seven minutes;
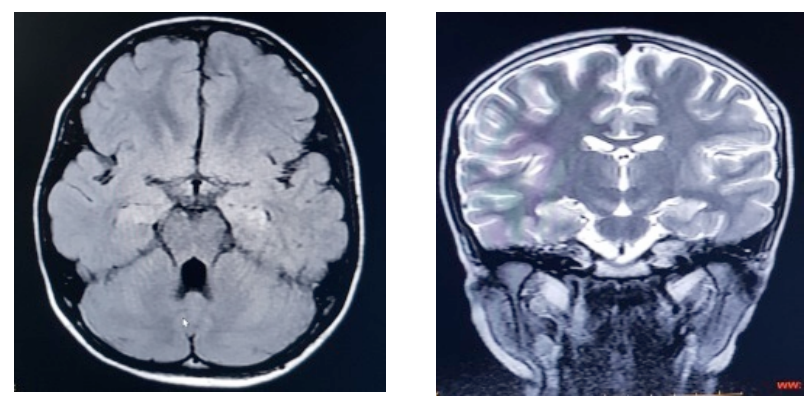

Figure1.

stamping it to be a case of status epilepticus treatment was started but consequently the patient landed into a case of refractory status epilepticus. Treatment was continued as per ILAE protocol. A guarded LP was done and CSF was sent for cytology, biochemistry, culture, gram stain, viral markers and autoimmune encephalitis panel. Over the next one day in view of persisting seizures dose of antiepileptic's gradually increased to its maximum level. By third day of admission, in view of persistent seizures midazolam infusion was gradually increased in view of super-refractory seizures. CSF cytology showed a total of 12 cells:all lymphomononuclear. Rest of the CSF paramteres were within normal limits. Inj methylprednisolone was added considering the case as autoimmune encephalitis. However, CSF autoimmune panel came negative. EEG was suggestive of abnormal awake record suggestive of seizure disorder originating from $\mathrm{B} / \mathrm{L}$ left side fronto-temporal foci generalisation. MRI showed FLAIR hyperintensity of hippocampal and parahippocampal gyrus (Mesial Temporal Sclerosis, Figure 1 and 2). Neuroimagings including CT and MRI/MRA of the brain revealed no intracranial or neurovascular lesions. This ruled out vascular or mass lesions as causes of her encephalopathy. HIV, herpes and syphilis screens were also negative. At this juncture, the possibility of an autoimmune phenomenon was entertained as all objective data were unrevealing. On day seven of admission due to persistent seizures IVIG was given at $2 \mathrm{gm} / \mathrm{kg}$ over five days in the line of autoimmune encephalitis. Mumps IgM and IgG was positive in CSF. Dramatic response was seen after 36-48 hours of IVIG administration. GCS

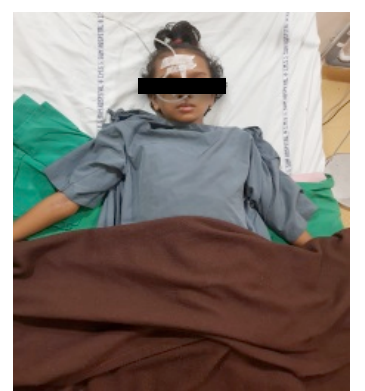

Figure 3.

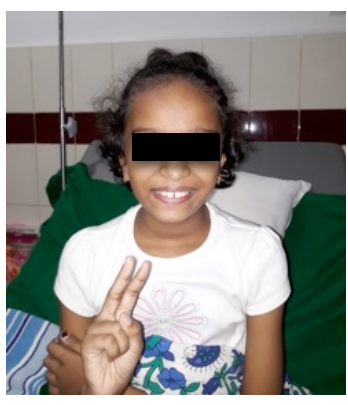

Figure 4. 
improved and seizures stopped. Child was discharged on antiepileptic drugs with regular follow-up advice (Figure-3). After three months repeat MRI revealed resolution of the previous abnormalities.

\section{DISCUSSION}

Encephalitis (inflammation of the brain parenchyma) is a common medical emergency in paediatrics. Failure to appropriately diagnose the correct aetiology of an encephalitic syndrome can lead to significant morbidity and mortality. Of infectious agents, viruses cause a significant proportion of encephalitis. Patients presenting with an encephalitic syndrome represent a diagnostic challenge. Estimated incidence of viral encephalitis in the UK, based on hospitalized patients from 1989 to 1998 , was approximately 1.5 per 100000 overall. ${ }^{3}$ Mesial temporal sclerosis, also known as hippocampal sclerosis, was found in our case following post mumps. Autoimmune encephalitis is the most common cause of temporal lobe epilepsy along with seizure refractoriness found at surgery. It is characterized histologically by a pattern of neuronal loss in which dentate gyrus becomes reorganized, leading to an aberrant hyperexcitable synaptic system. This is clinically manifested as recurrent seizures, or epilepsy. ${ }^{2,4}$ Apart from its association with the chronic nature of epilepsy, hippocampal sclerosis has shown to have an important role in internal cardiac autonomic dysfunction and unrecognised cause of cognitive decline, typically presenting with severe memory loss. ${ }^{5,7}$ The hallmark of mesial temporal sclerosis on MR imaging as in our case is an atrophic hippocampus associated with hyperintense signal on long-repetition-time sequences confined to the hippocampus. ${ }^{5,8} \mathrm{~A}$ thorough search, initially for an infectious aetiology and then for exclusion of underlying malignancy (tumour should be removed if found) was done in our case and she was started on intravenous immunoglobulin (IVIG) for presumed anti-NMDA receptor encephalitis. ${ }^{6,9}$ and showed dramatic response by second day of therapy. With the decline in uptake of the MMR vaccine there has been a recent increase in the number of mumps cases. Encephalitis is a recognised complication of mumps. It may present early by direct invasion following initial infection, or late as a post infectious (ADEM) event. ${ }^{10}$ Post mumps mesial temporal sclerosis with supra refractory status epilepticus in a child has not been previously reported. However, there is need of more number of cases and in depth molecular analysis to establish the exact pathophysiology of this rare phenomenon.

\section{CONCLUSIONS}

Autoimmune encephalitis is one of the commonest causes of super refractory status epilepticus in children. Important causes include HSV-1 \& 6, enterovirus and influenza virus. In our case we detected autoimmune encephalitis with super refractory status epilepticus following mumps parotitis. The excellent response to treatment in our patient also reiterates the need for prompt consideration, investigation and recognition of post mumps autoimmune encephalitis.

\section{REFERENCES}

1. Sloviter RS. Permanently altered hippocampus structure, excitability, and inhibition after experimental status epilepticus in the rat: the "dormant basket cell" hypothesis and its possible relevance to temporal lobe epilepsy. Hippocampus. 1991;1:41-66. https://doi.org/10.1002/hipo.23090

2. Asuman, OV, Serap, S, Hamit, A, Abdurrahman C. Prognosis of patients with mesial temporal lobe epilepsy due to hippocampal sclerosiS. Epilepsy Research. 2009;85(2):206-11. DOI:10.1016/j.eplepsyres.2009.03.001.

3. Davison KL, Crowcroft NS, Ramsay ME, Brown DW, Andrews NJ. Viral encephalitis in England, 1989-1998: what did we miss? Emerg Infect Dis. 2003;9:234-40. DOI:10.3201/eid2110.151125 
4. Jack CJ Jr, Sharbrough FW, Cascino GD, Hirschorn KA, O’Brien PC, Marsh WR. Magnetic resonance imagebased hippocampal volumetry: correlation with outcome after temporal lobectomy. Ann Neurol. 1992;31:138-46. DOI: 10.1002/ana.25411

5. Bronen RA, Fulbright RK, Spencer DD, Spencer SS, Kim JH, Lange RC, et al. Refractory epilepsy: comparison of MR imaging, CT, and histopathologic findings in 117 patients. Radiology. 1996 Oct;201(1):97-105. DOI:doi.org/ 10.1148/radiol.2020191160

6. Dalmau J, Gleichman AJ, Hughes EG, Rossi JE, Peng X, Lai M, et al. Anti-NMDA-receptor encephalitis: case series and analysis of the effects of antibodies, Lancet Neurol. 2008;7:1091-8. DOI:10.1136/bmj.327.7428.1364-b

7. Zarow, C; Weiner, MW; Ellis, WG; Chui, HC. Prevalence, laterality, and comorbidity of hippocampal sclerosis in an autopsy sample. Brain Behav. 2012;2(4):435-42. doi:10.1002/brb3.66.

8. Nelson PT, Schmitt FA, Lin Y, Abner EL, Jicha GA, Patel E, et al. Hippocampal sclerosis in advanced age: Clinical and pathological features. Brain. 2011 May;134(5):1506-18. doi: 10.1093/brain/awr053. PMID: 21596774

9. Aoki N, Murray ME, Ogaki K, Fujioka S, Rutherford NJ, Rademakers R, et al. Hippocampal sclerosis in Lewy body disease is a TDP43 proteinopathy similar to FTLD-TDP Type A. Acta Neuropathol. 2015 Jan;129(1):53-64. DOI: $10.1007 / \mathrm{s} 00401-014-1358-\mathrm{z}$.

10. Blumcke, I; Coras, R; Miyata, H; Ozkara, C. Defining Clinico-Neuropathological Subtypes of Mesial Temporal Lobe Epilepsy with hippocampal Sclerosis. Brain Pathology. 2012;22(3):402-11. DOI::10.1111/j. 1750-3639.2012.00583.x. 